\title{
Design of Intelligent Traffic Rules Planning System Based on Cellular Automation
}

\author{
Haoshen Lin, Gang Liu, Bing He, Xiaojun Yang, Chen Hu \\ Department of Aerospace Engineering, Research Institute of Hi-Tech, XI'AN 710025 \\ E-mail: linhaoshen1@163.com
}

Keywords: Traffic Rules Analysis, Intelligent System, Cellular Automation

\begin{abstract}
In order to analyze the validity and scientificity of the traffic rules, we design an intelligent traffic rules analysis system. According to the complexity of traffic flow, we adopt a revised cellular automation to establish corresponding simulation model, in which each vehicle is regarded as a cellular. As the position and speed of each vehicle in the traffic flow are all continual, we alter the conventional cellar space and adjacent zone. We propose and quantify the indexes, including traffic volume, safety and level of service (LOS) of the road to evaluate the performance of traffic rules, and the rationality of road speed limits is analyzed. The results of simulation show that safety and traffic volume are contradicted. Hereafter, we propose two methods to balance the two indexes and provide recommendations for the traffic administrative branches. Meanwhile, the simulation results show the weakness of low road occupancy in light traffic. In terms of the weakness, we propose a new rule, under which vehicles with different speeds travel on different lanes and the speed limits for different lanes are different. Simulation results show that only in light traffic this rule is efficient.
\end{abstract}

\section{Introduction}

Currently, in countries where driving automobiles on the right is the rule (that is, USA, China and most other countries except for Great Britain, Australia, and some former British colonies)[1], multi-lane freeways often employ a rule that requires drivers to drive in the right-most lane unless they are passing another vehicle, in which case they move one lane to the left, pass, and return to their former travel lane[2].

Because of the nonlinear, time-varying and strong stochasticity and complexity of urban traffic system, it is difficult to describe and control the system accurately by establishing traditional prediction model[3]. In order to improve the traffic efficiency at the intersection, the local government to increase investment to establish a more complete road hardware facilities, while research institutes in the modern control theory based on the study of the use of artificial intelligence methods to further improve vehicle traffic efficiency[4] [5].

Many scholars have used a variety of methods to study the characteristics of traffic flow, traffic flow forecast, traffic capacity, traffic flow delay, and achieved some results. From the aspects of timing[6], induction and adaptive control[7], three kinds of control methods are studied separately, and point control is proposed for the reality of urban traffic vehicle, Wire control and surface control[8] [9].

Our goal is to build and analyze a mathematical model to analyze the performance of this rule in light and heavy traffic. In our dual-lane model, we take fully consideration to the tradeoffs between traffic flow and safety, the role of under- or over-posted speed limits, and level of service.

This dissertation investigates in Intelligent Traffic.

Intelligent Traffic Rules Analysis System. We intend to analyze the performance of a specific rule in light and heavy traffic. In fact, the traffic flow change of the freeway is an evolution process of a complex system.

Model of Cellular Automation. In our model, each cellular represents an individual vehicle. We assume that each cellular possesses its respective attributes (including the vehicle type, the 
desired speed, the driver's responding time, and the actual travel speed, etc.) and behaviors (including free travel, passing, following and lane-moving, etc.)

The conventional cellular space (shown in Fig. 1) is grid space, in which the cellular are distributed and their moving is discrete due to the restriction of the grid space. However, in our model, the travel speed and travel distance mileage are continual. So we set the cellular space as partially continual space.

The conventional adjacent zone of a cellular is illustrated in grey, as shown in left of Fig.1. While in our model, the cellular space and its adjacent zone are shown in right of Fig 1, as the moving space of the cellular is restricted by the number of lanes.

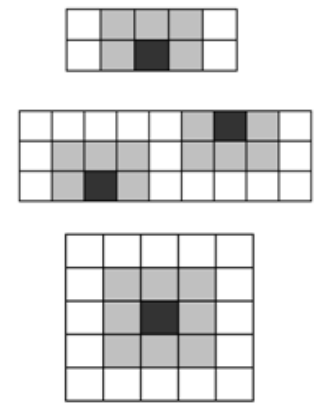

Fig.1 Conventional cellular space and its adjacent zone

Design of Intelligent Tracffic Rules Analysis System. Considering the computer programs and organization structure of the model, we subdivide the simulation model into four modules: input module, functional module, simulation module and output module, as shown in Fig.2.

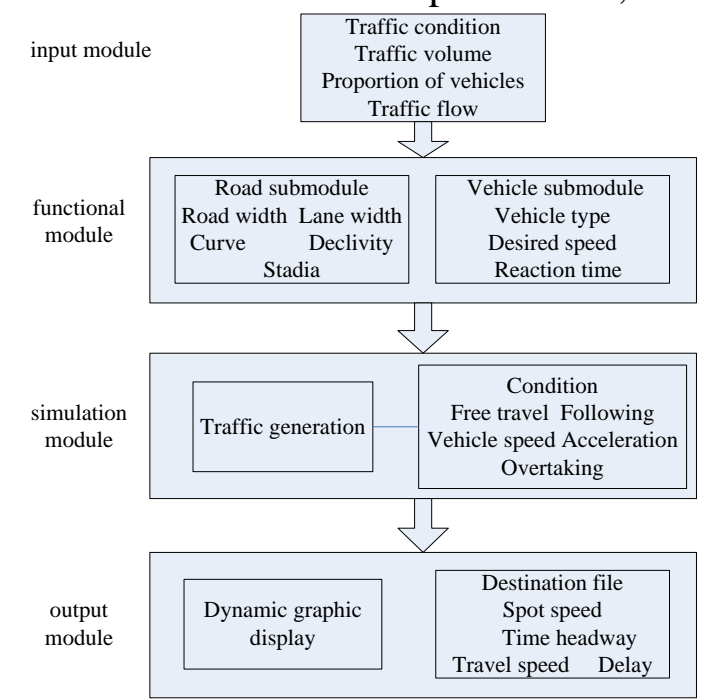

Fig.2. The organization structure of the simulation model

1) Input module

The upstream traffic pressure $\mathrm{X}$ : we regard the incoming traffic flow entering the target freeway section as a Poisson flow, the parameter $\mathrm{X}$ indicates the mathematical expectancy of incoming vehicle number with a specific time unit. To some extent, its value can reflect the stress of the upstream incoming vehicles. Therefore, we define the parameter as upstream traffic pressure.

The proportion of the vehicle type refers to the ratio of slow vehicles in all vehicles, which is a constant.

2) Functional module

Road sub-module: road width, lane width, speed limit

As the module only focuses on the influence of a specific rule, so the infrastructure of the road is not taken into consideration.

Speed limits include maximum speed limit $V_{h}$ and minimum speed limit $V_{l}$.

Vehicle sub-module: vehicle type, desired speed, driver’s responding time, braking time 
The desired speed varies because of the different driving habits. In our model, we assume that the desired speeds of the two vehicle types accord with normal distribution. When the speed limit is within the range from $60-120 \mathrm{~km} / \mathrm{h}$, the possibility density curve of the slow vehicle speed $\mathrm{N}\left(\mathrm{u}_{\mathrm{l}}, \sigma_{\mathrm{l}}\right)$ and the fast vehicle speed $\mathrm{N}\left(\mathrm{u}_{\mathrm{h}}, \sigma_{\mathrm{h}}\right)$ is shown in Fig. 3.

$$
\mathrm{u}_{\mathrm{l}}=\frac{\mathrm{v}_{\mathrm{h}}+\mathrm{V}_{1}}{2}+\frac{\mathrm{v}_{\mathrm{h}}-\mathrm{V}_{\mathrm{l}}}{4}, \mathrm{u}_{\mathrm{h}}=\frac{\mathrm{v}_{\mathrm{h}}+\mathrm{V}_{1}}{2}-\frac{\mathrm{v}_{\mathrm{h}}-\mathrm{V}_{1}}{4}, \sigma_{l}=\frac{1}{12}\left(V_{h}-V_{l}\right), \sigma_{h}=\frac{1}{12}\left(V_{h}-V_{l}\right)
$$

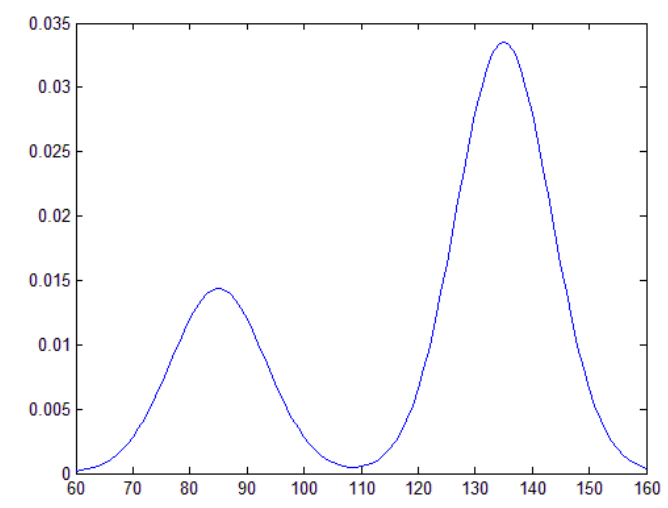

Fig.3 The possibility density curve of the slow vehicle speed

We simply the driver's responding time and the braking time and assume the responding time as $1.2 \mathrm{~s}$ and the braking time as 1-8s.

3) Simulation module

Driver's behavior pattern: free travel, passing, following and lane-moving.

Considering the driver's behavior pattern on freeway, we can illustrate the driver's behavior selection tree and behavior process in Fig. 4.
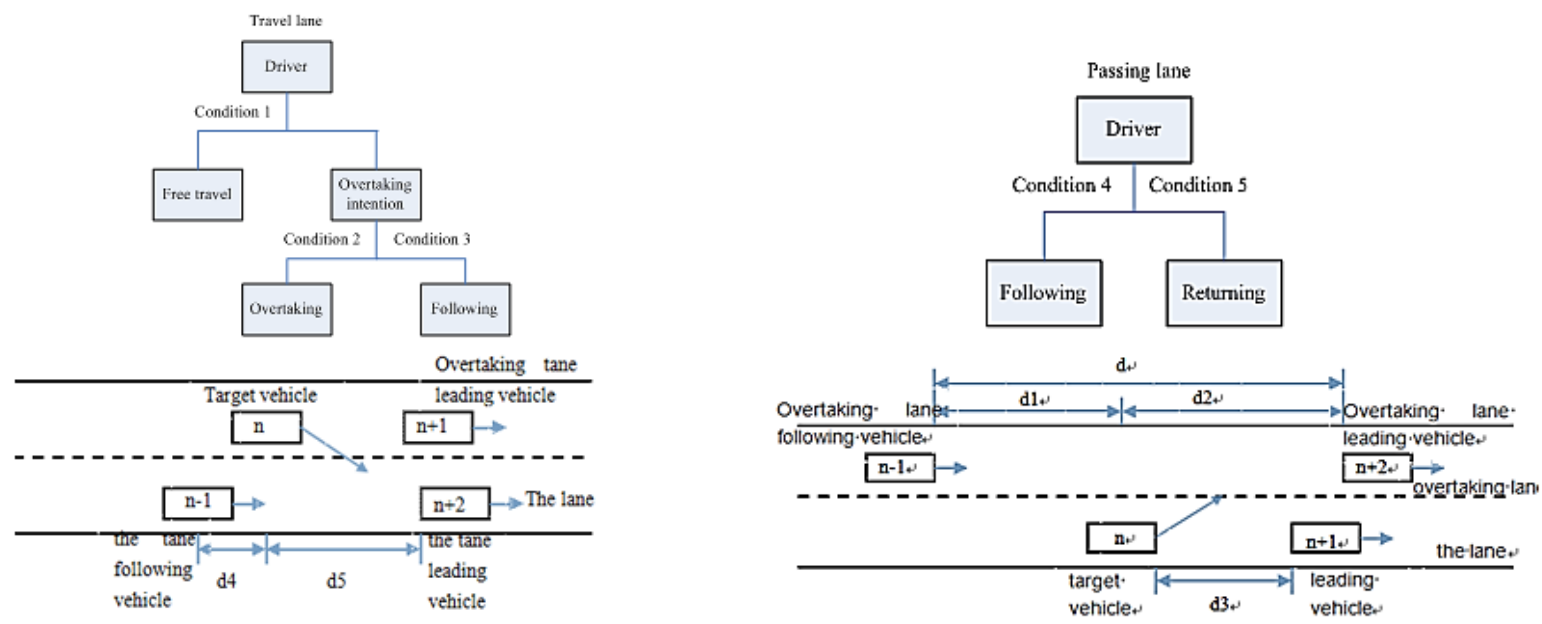

Fig.4 the driver's behavior selection tree and behavior process

In traveling, the driver needs to decide his behavior according to the road condition. On the travel lane, when passing condition is satisfied, the driver may desire to pass and then chooses to pass or follow based on the lane-moving condition.

On the passing lane, the driver may choose to move to the travel lane or follow on the passing lane based on the lane-returning condition.

$$
\text { Objective vehicle }==\left\{n, j, m, p, V_{e}, V_{a}\right\}
$$

Where, variables include: $n$, which is the series number of the objective vehicle on the freeway, $j$, which is series number of the lane ( $\mathrm{j}=1$ refers to the passing lane and $\mathrm{j}=2$ refers to the travel lane), $p$, which is horizontal position of the objective vehicle in the cellular space $(0<p<1000)$, and $V_{a}$, which is the actual speed of the objective vehicle; 
Constants include: $m$, which is the vehicle type of the objective vehicle $(\mathrm{m}=1=$ refers to fast vehicle and $m=2$ refers to slow vehicle) and $V_{e}$, which is desired speed of the objective vehicle.

In driving of the objective vehicle, to better describe the driving safety of the vehicle, we assume that the passing time interval $\mathrm{T}_{\text {pass }}=20 \mathrm{~s}$, the safe time interval $\mathrm{T}_{\text {safe }}=3 \mathrm{~s}$ (the response time is $1.2 \mathrm{~s}$ and the braking time is 1.8s). Then the distance of passing $\mathrm{d}_{\text {pass }}=20 * \mathrm{~V}_{\mathrm{r}}$, and the safe distance $\mathrm{d}_{\text {safe }}=3 * V_{\mathrm{a}}$, where $\mathrm{V}_{\mathrm{r}}$ is the relative speed of the objective vehicle and the preceding vehicle.

(1) Passing condition

Condition 1: When the distance between the objective vehicle and the preceding vehicle exceeds both $d_{\text {pass }}$ and $d_{\text {safe }}$, the driver may have the desire to pass.

(2) Lane-moving condition

Condition 2: Both the preceding vehicle and the following vehicle on the passing lane are farther away from the objective vehicle.

(3) Lane-returning condition

Condition 3: Both the preceding vehicle and the following vehicle on the travel lane are farther away from the objective vehicle

The driver's behavior patterns under the keep-right-except-to-pass rule:

(1) Travel freely

(2) Follow on the travel lane

$$
\left\{\begin{array}{l}
j_{t+\Delta t}=j_{t} \\
p_{t+1}=p_{t}+v_{a(t+\Delta t)} \Delta t \\
v_{a(t+\Delta t)}=v_{a t}
\end{array}\right.
$$

$$
\left\{\begin{array}{l}
j_{t+\Delta t}=j_{t} \\
p_{t+\Delta t}=p_{t}+\left(v_{a}-c_{1}\right) \Delta t
\end{array}\right.
$$

(3) Pass

(4) Follow on the passing lane

$$
\left\{\begin{array}{l}
j_{t+\Delta t}=1 \\
p_{t+\Delta t}=p_{t}+v_{e} \Delta t \\
v_{a(t+\Delta t)}=v_{e}
\end{array}\right.
$$

(5) Return to the travel lane

$$
\left\{\begin{array}{l}
j_{t+\Delta t}=j_{t} \\
v_{a(t+\Delta t)}=v_{a t}+c_{2} \\
p_{t+\Delta t}=p_{t}+v_{a(t+\Delta t)} \Delta t
\end{array}\right.
$$

4) Output module

Actual vehicle speed, average speed of each vehicle. Dynamic graph display

Level of service. Highway Capacity Manual 2000 (HCM 2000) of America defined the Level of Service (LOS) as "a quality measure describing operational conditions within a traffic stream, generally in terms of such service measures as speed and travel time, freedom of maneuver, traffic interruption, and comfort and convenience.” (HCM 2000: 25)

HCM 2000 proposed a new method to calculate the levels of service of urban roads. The main thought is as follows: based on the general average speed $\left(\mathrm{V}_{\mathrm{a}}\right)$ of a vehicle and free flow speed $\left(\mathrm{V}_{\mathrm{f}}\right)$, the LOSs of the whole road and each road section are determined, where, the free flow speed is the theoretical vehicle speed when the density is zero[1] or the travel speed which is not disturbed by other vehicles and freely chosen by the driver according to his subjective desire[2]. 
Based on HCM 2000, the levels of service for road transportation are classified into 6 levels:
(A) Free Flow Traffic (LOS $>90 \%$ ).
(B) Steady Traffic $(90 \%>\operatorname{LOS}>70 \%)$.
(C) Steady Traffic but Limited (70\% $>$ LOS $>50 \%)$.
(D) Steady Traffic at High Density (50\%>LOS $>40 \%)$.
(E) Traffic at Saturation (40\%>LOS $>33 \%)$.
(F) Congestion ( $\mathrm{LOS}<33 \%)$.

\section{Simulation Result Analysis}

The influences of the upstream traffic pressure (UTP) on road performance. As shown in Fig. 5(a), under the condition of speed limits being $60-120 \mathrm{~km} / \mathrm{h}$, we assume all vehicles travel under the rule. When the upstream traffic pressure reaches or exceeds 4000 veh./h, the traffic volume reaches the maximum value 1800 veh./h and keeps steady. Then the traffic flow can be regarded to be at saturation. We assume that when the traffic volume reaches or exceeds 1800 veh./h, the traffic flow is heavy. When the upstream traffic pressure increases to $600 \mathrm{veh} . / \mathrm{h}$, the traffic volume increases to 570 veh./h. But from the perceptive of LOS(Fig. 5(b)), the road LOS is degraded to be LB from LA, and the freedom of maneuver is slightly influenced. We assume that when the traffic volume is lower than $570 \mathrm{veh} . / \mathrm{h}$, the traffic flow is light.
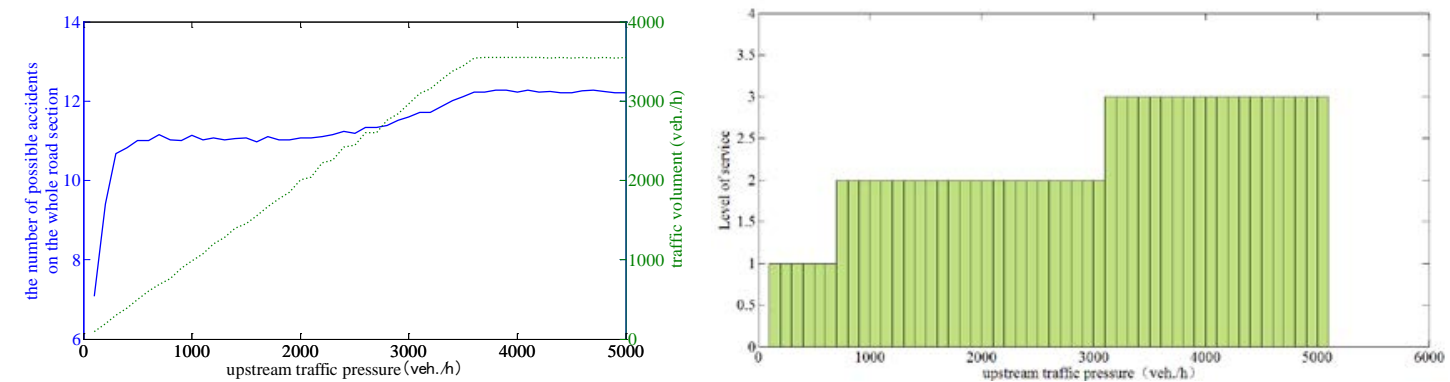

Fig.5(a) The influences of the UTP on the traffic volume Fig.5(b) The influences of the UTP on LOS

Analysis concerning speed limits. In order to judge whether the speed limits are too high or too low in light or heavy traffic, we set maximum and minimum speed limits as independent variances respectively and the upstream traffic pressure as a constant, to observe the corresponding road performances. Here, the number of possible accidents on the whole road section can be simplified to be the accident rate.

Simulation condition is set as follow:

Case 1: When the upstream traffic pressure is $600 \mathrm{Veh} . / \mathrm{h}$, and the minumum speed limit is fixed to be $60 \mathrm{~km} / \mathrm{h}$ while the maxium speed limit varies from 60 to $260 \mathrm{~km} / \mathrm{h}$.

Case 2: When the upstream traffic pressure is $600 \mathrm{Veh} . / \mathrm{h}$, and only the maxium speed limit is fixed to be $120 \mathrm{~km} / \mathrm{h}$ while the minimum speed limit varies from 0 to $120 \mathrm{~km} / \mathrm{h}$.

Case 3: When the upstream traffic pressure is $4000 \mathrm{Veh} . / \mathrm{h}$, and the minumum speed limit is fixed to be $60 \mathrm{~km} / \mathrm{h}$ while the maxium speed limit varies from 60 to $260 \mathrm{~km} / \mathrm{h}$.

Case 4: When the upstream traffic pressure is $4000 \mathrm{Veh} . / \mathrm{h}$, and the maximum speed limit is fixed to be $160 \mathrm{~km} / \mathrm{h}$ while the minimum speed limit varies from 0 to $160 \mathrm{~km} / \mathrm{h}$.

To simplify the analysis process, we just analyze the simulation resulet under the case 3.

When the upstream traffic pressure is $4000 \mathrm{Veh} . \mathrm{h}$, and the minumum speed limit is fixed to be $60 \mathrm{~km} / \mathrm{h}$ while the maxium speed limit varies from 60 to $260 \mathrm{~km} / \mathrm{h}$, we can observe from Fig. 6(b) that the LOS will upgrade from Level C to Level B. The main reason is that when the maximum speed limit reaches $160 \mathrm{~km} / \mathrm{h}$, the relatively congested traffic becomes relatively unobstructed. But hereafter, the number of possible accidents may soar up. Therefore, it is reasonable to set the maximum speed limit as $160 \mathrm{~km} / \mathrm{h}$ in such situation. 


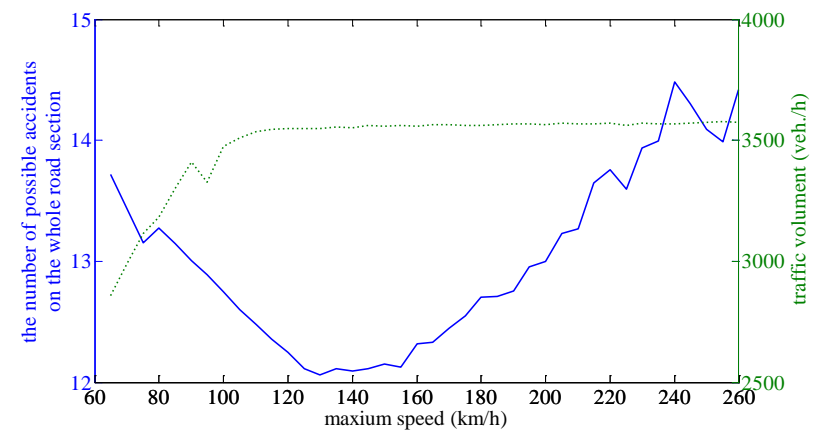

Fig.6(a) The influences of the UTP on the traffic volume

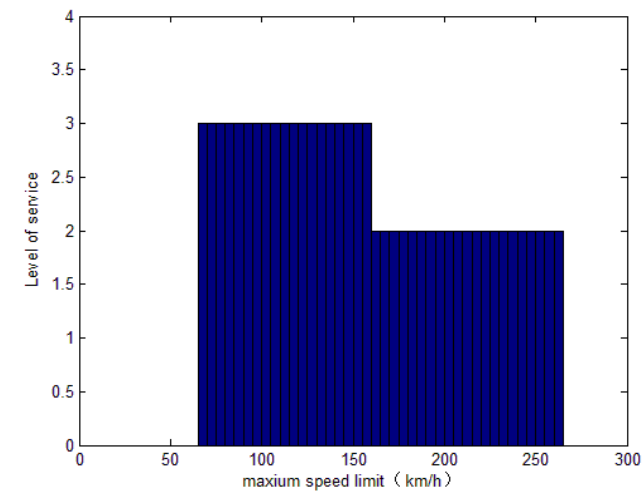

Fig.6 (b) The influences of the UTP on LOS

Taking the simulation results in light and heavy traffic into comprehensive consideration, as for a freeway section with $60-160 \mathrm{~km} / \mathrm{h}$ limits, we regard that the maximum speed limit is too high and may be reduced to $120 \mathrm{~km} / \mathrm{h}$ if the traffic is light. Similarly, if the traffic is heavy, we regard that the minimum speed limit is too low and may be increased to $120 \mathrm{~km} / \mathrm{h}$. In this way, the vehicles are at free flow with relatively high safety.

\section{Conclusions}

With the development of modern transportation and the increase of traffic pressure, the two-lane freeways cannot meet the requirements. Currently, three-lane freeways have been widely used. Therefore, we also carry out the simulation of three-lane freeway under various rules. And the rules include:

(1)The exterior lane is the travel lane, the middle lane is pass lane I and the interior lane is pass lane II, vehicles are only allowed to travel on the travel lane if they don't pass and vehicles are only permitted to pass from the left.

(2)The exterior and middle lanes are travel lanes, and the interior lane is the pass lane. Vehicles are only permitted to pass from the left.

(3)The exterior lane is the slow lane, the middle lane is the fast lane and the interior lane is the pass lane. Slow vehicles are not allowed to travel on the pass lane.

(4)The exterior lane is the slow lane, the middle lane is the pass lane and the interior lane is the fast lane.

(5)The exterior lane is the fast lane, the middle lane is the pass lane and the interior lane is the slow lane.

In the rules above, the speed limits of fast lane and slow lane are set as $90-120 \mathrm{~km} / \mathrm{h}$ and $60-90$ $\mathrm{km} / \mathrm{h}$ respectively. Considering the evaluation indexes, we choose the third rule as the optimal rule for three-lane freeway.

\section{References}

[1] Salki Ami, Ida Tomoyuki (Trans. Shao Chunfu etc). Transportation Engineering [M]. Beijing: The People's Transportation Press,1994:117

[2] Li Jiang, Xu Guang-yong, Liu Li. An analysis of free flow speed of the two-lane road[J]. Journal of Liaoning Provincial College of Communication, 2003, 5(4):6-7/22.

[3] Li Jiang, Xu Guang-yong, Liu Li. An analysis of free flow speed of the two-lane road[J]. Journal of Liaoning Provincial College of Communication, 2003, 5(4):6-7/22.

[4] Wang hai yan,Study on the highway traffic safety evaluation system 2009.

[5] A Evaluation Method of Intelligent Transport System Socioeconomic Impact ZhiCai Jun. page .83.Beijing: Tsinghua Press.2008.8. 
[6] Smith RD. THE DYNAMICS OF INTERNET TRAFFIC: SELF-SIMILARITY SELF-ORGANIZATION, AND COMPLEX PHENOMENA [J] .ADVANCES IN COMPLEX SYSTEMS, 2011, 14 (6):905-949.

[7] Gershenson C, Rosenblueth DA. Adaptive self organization vs static optimization A qualitative coordination [J]. KYBERNETES comparison in traffic light 2012, 41 (3) 386-340.

[8] De Meer Hermann, sterbenz james P G. Self-organising systems [M]. Euron; First International Workshop,2006.

[9] Gao Ziyou, Long Jiancheng, Li Xingang. Congestion propagation law and dissipation control strategies for urban traffic[J]. University of Shanghai for Science and Technology, 2011, 33(6):701-708. 